East African Medical Journal Vol. 85 No. 6 June 2008

CORRELATION OF CLINICAL DATA, ANATOMICAL SITE AND DISEASE STAGE IN COLORECTAL CANCER

H.S. Saidi, BSc., MBChB, Senior Lecturer, Department of Human Anatomy, D. Karuri, Medical Student and E.O. Nyaim, MBChB, MMed, Department of Surgery, School of Medicine, University of Nairobi, P.O. Box 19767-00202, Nairobi, Kenya

Request for reprints to: Dr. H.S. Saidi, Department of Human Anatomy, School of Medicine, University of Nairobi, P.O. Box 30197-00100, Nairobi, Kenya

\title{
CORRELATION OF CLINICAL DATA, ANATOMICAL SITE AND DISEASE STAGE IN COLORECTAL CANCER
}

\author{
H.S. SAIDI, D. KARURI and E.O. NYAIM
}

\begin{abstract}
Objective: To evaluate the colorectal cancer clinical data with respect to the anatomical location and stage of disease.

Design: Retrospective observational study.

Setting: Kenyatta National Hospital (KNH), Nairobi, Kenya.

Subjects: Two hundred and fifty three tumours were categorised as right colonic (RCC), left colonic (LCC) and rectal (RC) lesions. The distribution of symptoms (rectal bleeding, tenesmus, change in bowel habits, abdominal pain, intestinal obstruction, rectal mass), anaemia, transfusion requirement, and the Dukes' stages were compared for right colon, left colon and rectal tumours.

Results: There were 54 RCC, 59 LCC, 140 RC lesions. Patient delay from onset of symptom(s) to presentation was a mean of $26.6 \pm 43,20 \pm 25$ and $33.7 \pm 42$ weeks for right, left and rectal lesions respectively $(\mathrm{p}=0.092)$. The proportion of patients presenting with rectal bleeding was $21 \%, 44 \%$ and $79 \%$ for RCC, LCC and RC lesions, respectively. The prevalence of intestinal obstruction was $14.8 \%, 27.1 \%$ and $43.6 \%$ in right, left and rectal lesions, respectively. The haemoglobin levels were significantly lower for right sided lesions ( $p=0.05$ for right colon/rectum pair; $p=0.059$ for right colon/ left colon pair). The sites of the lesions had no relationship to the stage of disease at presentation. Conclusion: In patients with colorectal cancer, the duration of symptoms was prolonged irrespective of the anatomical sub-sites. Symptoms were evenly distributed across the anatomical regions except for bleeding and obstruction which predominated in rectal and left colon cancers respectively. This underlines the need for early investigations in patients with rectal bleeding, change of bowel habit, intestinal obstruction and anaemia.
\end{abstract}

\section{INTRODUCTION}

Colorectal carcinoma (CRC) is increasingly being reported in the developing countries (1). In these countries, screening is unusual and the diagnosis is made after the onset of symptoms. Because the stage of the disease at presentation is the most important prognostic factor in CRC, early symptom investigation and diagnosis is logical. But, even where the disease prevalence is high, the awareness of colorectal cancer and its symptoms is low (2). Further, the non-specific nature of the symptoms ensures that the search for those with high predictive values for colorectal cancer continues. These symptoms may be associated with the anatomic sites of distribution and in turn, the stage of the cancer. Colorectal cancer in Kenya is predominantly a left sided disease (3). We hypothesized that patients with left colonic cancer will present early with obstructive symptoms due to more formed stools and constricting nature of left sided tumour lesions. In addition, the bleeding in rectal tumours will dictate earlier presentation. 
This review evaluates the CRC clinical data with respect to the anatomical location in 253 patients treated at Kenyatta National Hospital in Nairobi.

\section{MATERIALS AND METHODS}

Design: This was a retrospective review of medical records, analysing the clinical presentation of colorectal carcinoma in relation to stage and site of the lesion.

Setting: Kenyatta National Hospital (KNH), a teaching and a national referral hospital. The $\mathrm{KNH}$ research and ethics committee approved the study.

Methods: Two hundred and fifty medical records of patients treated between 1993 and 2005 were retrieved. The site distributions were categorised as right colonic (RCC), left colonic (LCC) and rectal tumours (RC). Demographic data (age, sex) and the distribution of symptoms (rectal bleeding, tenesmus, change in bowel habits, abdominal pain, intestinal obstruction, rectal mass), anaemia, transfusion requirement, and the Dukes' stages were compared for right colon, left colon and rectal tumours. Only records with confirmed histopathological reports of colorectal carcinoma diagnoses were reviewed.

\section{Definitions of variables:}

Right-sided: Lesions arising from the caecum, ascending colon, hepatic flexure or transverse colon.

Left-sided: Lesions arising from splenic flexure, descending colon or sigmoid colon.

Dukes' A: Growth confined to the wall,
Dukes ' $B$ : Growth to the serosa and beyond but no nodal involvement

Dukes' C: Growth beyond serosa and involving nodal basins

Dukes 'D: Metastatic disease.

Statistical analysis: The data were analysed using the computer statistical program SPSS (version 11.5 for Windows, SPSS Inc., Chicago, Ill). The one way analysis of variance (ANOVA) was used to compare continuous variables across the three groups. Post hoc analyses were performed to determine pairs with significant differences. Statistical significance was set at $\mathrm{p}<0.05$.

\section{RESULTS}

There were 54 RCC, 59 LCC and 140 RC lesions. Six cases had indeterminate sites. Confirmation of diagnosis was based on biopsy and/or resection specimen histopathology reports for all the lesions. Males predominated in all the three anatomical sites (65\% for RCC, 59\% for both LCC and RC). The modal age group at presentation was 41-50 years for all the sites (Figure 1).

The respective mean ages of $49.98,47.17$ and 50.67 years for the three groups were statistically similar $(\mathrm{p}=0.373)$.

Patient delay from onset of symptom to presentation was a mean of $26.6 \pm 43,20 \pm 2.25$ and $33.7 \pm 42$ weeks for right, left and rectal lesions, respectively ( $\mathrm{p}=$ 0.092). The proportion of patients presenting with rectal bleeding was $21 \%, 44 \%$ and $79 \%$ for RCC, LCC and RC lesions, respectively (Table 1 ). There was no

Figure 1

Age distrinution of colorectal cancer, $\mathrm{KNH}$

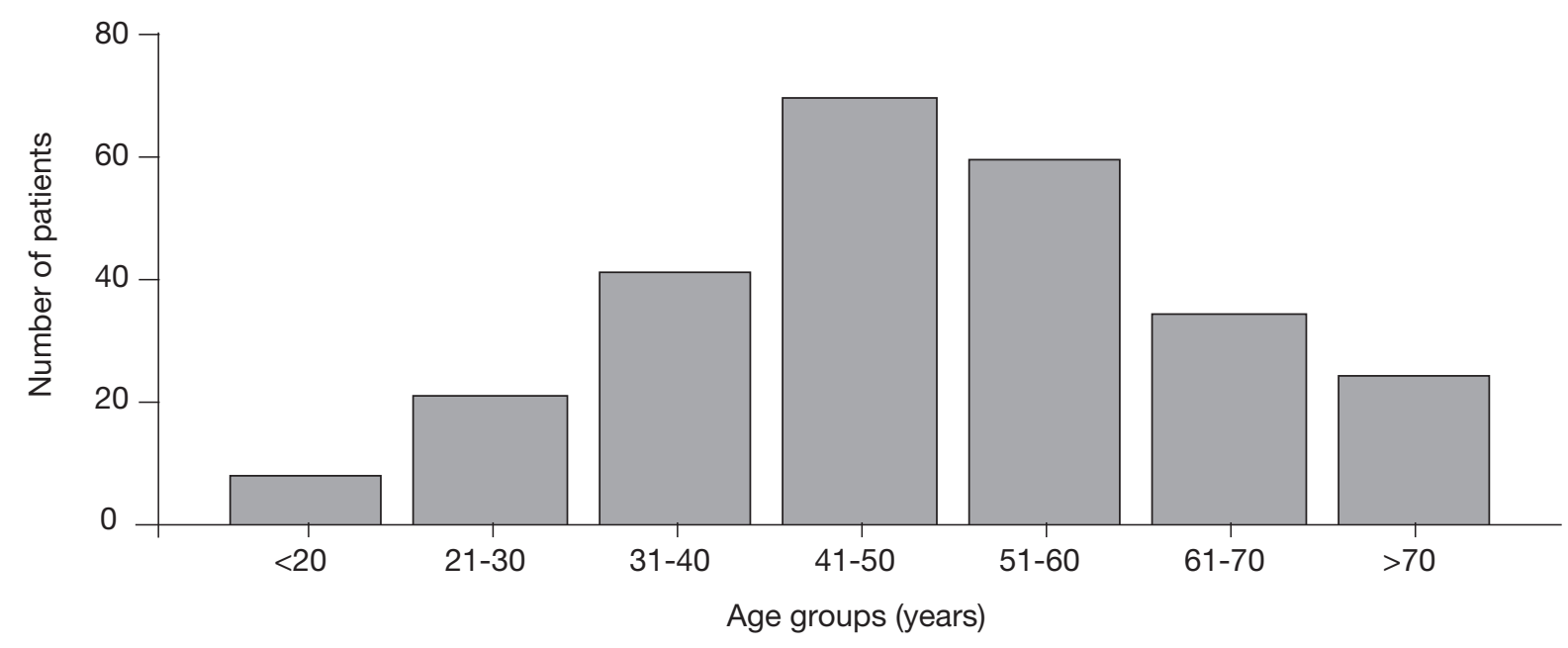


Table 1

Symptomatology of colorectal cancer at KNH

\begin{tabular}{lcccccc}
\hline & \multicolumn{2}{c}{ Right colon } & \multicolumn{2}{c}{ Left colon } & \multicolumn{2}{c}{ Rectum } \\
Symptom & No. & $(\%)$ & No. & $(\%)$ & No. & $(\%)$ \\
\hline Rectal bleeding & 11 & 21.2 & 25 & 43.9 & 107 & 79.3 \\
Tenesmus & 6 & 11.5 & 9 & 16.1 & 41 & 30.8 \\
Abdominal pain & 40 & 76.9 & 48 & 84.2 & 81 & 60.0 \\
Intestinal obstruction & 8 & 14.8 & 16 & 27.1 & 61 & 43.6 \\
Mucus discharge & 0 & & 4 & 7.0 & 33 & 24.3 \\
Mass on DRE* & 2 & 3.8 & 16 & 28.6 & 122 & 91.0 \\
Changed bowel habit & 33 & 63.5 & 48 & 85.7 & 89 & 66.4 \\
\hline
\end{tabular}

* Digital rectal examination

recorded mucus discharge for right sided lesions while left and rectal lesions recorded $7 \%$ and $24 \%$ respectively. Tenesmus was reported in 31\% for rectal lesions; right and left sided lesions recorded $12 \%$ and $16 \%$ respectively. Eighty six per cent of patients with left colon lesions reported a change in bowel habit. This proportion was $77 \%$ and $60 \%$ for RCC and RC. Abdominal pain was reported for $84 \%, 77 \%$ and $60 \%$ of patients with LVCC, RCC and RC lesions (Table 1). The tumour was palpable on digital rectal examination (DRE) in $91 \%$ and $29 \%$ of rectal lesions and left colon lesions respectively. The prevalence of intestinal obstruction was $14.8 \%, 27.1 \%$ and $43.6 \%$ in right, left and rectal lesions respectively.

The mean haemoglobin level at presentation was 10.5, 11.6 and $11.4 \mathrm{~g} / \mathrm{dl}$ for right, left and rectal lesions, respectively. The haemoglobin levels were significantly lower for right sided lesions $(p=0.05$ for right colon/ rectum pair; $p=0.059$ for right colon/left colon pair). Most patients received transfusion peri-operatively $(62.2 \%$ RCC, $61.8 \%$ LCC, 70.6\% RCC).

Seventy one per cent of patients with rectal lesions presented at Dukes' stages C and D. In comparison, $78.6 \%$ of RCC and $60.0 \%$ of LCC patients presented at Dukes' C and D.

\section{DISCUSSION}

Early diagnosis influences the survival of patients diagnosed with colorectal cancer. The present data indicate prolonged delays from the onset of symptoms to presentation for all the anatomical sub-sites. The relatively better result for the left colon is anticipated. Constricting tumours are more likely to characterise the descending colon which also contains more formed contents (4). Although certain patient, geographical and physician factors may have contributed to the diagnostic delay $(5,6)$, there is need to develop early referral guidelines based on symptomatology.

Rectal bleeding was the commonest symptom for rectal cancer in the current study. The challenge with rectal bleeding is the myriad of benign lesions that may be responsible in the primary care setting (7-10). In one survey for example, a prevalence of rectal bleeding in the UK population stood at $14 \%(7,8)$. Previous studies have however, indicated that rectal bleeding can reasonably predict colorectal cancer, especially when reported in older patients and in combination with change in bowel habits or abdominal pain $(9,10)$. Thus, in the UK, patients are referred for colorectal cancer investigations when they present with rectal bleeding and changes in bowel habits (7). Although there is data to suggest lower risk in the general population when bleeding is combined with local symptoms of painful defecation and pruritus, several cancer series have highlighted the significance of these latter symptoms in colorectal cancer patients $(11,12)$. It would appear therefore, that it may be worth investigating rectal bleeding in all cases.

The assessment of change in bowel habits may be difficult to document because of patient variability (6). The present series indicates a poor site localization for this symptom. In accounts from UK, changes towards increased stool frequency and diarrhoea are reportedly more predictive of cancer (predictive value 8\%) than constipation (predictive value 1.4\%) (13). The converse may be true for our data. The disease is predominantly left-sided and $27 \%$ and $43.6 \%$ of colon and rectal lesions presented with intestinal obstruction respectively. We contend that these obstructive symptoms should dictate early referrals to impact positively on survival. 
Patients with left-sided obstructing cancer due to advanced diseases have worse outcomes compared to non-obstructing tumours (14).

The secondary effects of colorectal cancer may also be important pointers towards fast-tracking investigations for earlier diagnosis. Our results show significantly lower haemoglobin levels for RCC lesions. These right-sided tumours tend to be larger and so bleed easily as opposed to left-sided tumours which are smaller and obstructing in nature. Thus, the threshold for investigating for colon cancer should remain low for adult patients presenting with anaemia $(15,16)$.

Abdominal pain was also a common but nonspecific symptom in this study. This is true of several other studies. In one account, the proportion of this symptom in patients referred for suspected colorectal cancer was $54 \%$ with a positive predictive value of only $2.7 \%$ (10). The physical finding of rectal masses underscores the utility of complete physical assessment during abdominal physical examination. In hospital series of colorectal cancer, abdominal and rectal masses are reported in 4-6\% and $24-50 \%$ of patients, respectively (15-17).

In conclusion, symptoms of colorectal cancer are not specific to anatomic sites. The onset of colorectal cancer symptoms is associated with late presentation in all anatomical sub-sites. More studies on the predictive powers for single or combination of symptoms are needed. Early colorectal investigations for the patients with rectal bleeding, change of bowel habits and anaemia may improve the outlook.

\section{ACKNOWLEDGEMENTS}

To the KNH management for permission to carry out this study. To those anonymous medical students at the University of Nairobi who prevailed upon us to undertake this study.

\section{REFERENCES}

1. Ashenafi, S. The frequency of large bowel cancer as seen in Addis Ababa University, Pathology Department. Ethiop. Med. J. 2000; 38: 277-282.

2. Sontag, S.J., Durczack, C., Aranha, G.V., et al. Foecal occult blood screening for colorectal cancer in a veterans administration hospital. Amer. J. Surg. 1983; 145: 89-94.

3. Saidi, H., Nyaim, E., Githaiga, J., et al. Colorectal cancer surgery in Kenya, 1993-2005. World J. Surg. 2008; 32: 217-223.
4. Robertson, R., Campbell, N.C., Smith, S., et al. Factors influencing time from presentation to treatment of colorectal and breast cancer in urban and rural areas. Brit. J. Cancer. 2004; 90: 1479-1485.

5. Campbell, N.C., Elliott, A.M., Sharp, L., et al. Impact of deprivation and rural residence on treatment of colorectal and lung cancer. Brit. J. Cancer. 2002; 87: 585-590.

6. Hamilton, W. and Sharp, D. Diagnosis of colorectal cancer in primary care: the evidence base for guidelines. Fam. Prac. 2004; 21: 99-106.

7. Crosland, A. and Jones. R. Rectal bleeding: prevalence and consultation behaviour. Brit. Med. J. 1995; 311: 486-488.

8. Metcalf, J.V., Smith, J., Jones, R., et al. Incidence and causes of rectal bleeding in general practice as detected by colonoscopy. Brit. J. Gen. Pract. 1996; 46: 161-164.

9. Norrelund, N. and Norrelund, H. Colorectal cancer and polyps in patients aged 40 years and over who consult a GP with rectal bleeding. Fam. Pract. 1996; 13: $160-165$.

10. Selvachandran, S., Hodder, R., Ballal, M., et al. Prediction of colorectal cancer by a patient consultation questionnaire and scoring system: a prospective study. Lancet. 2002; 360: 278-283.

11. Curless, R., French, J., Wilimas, GV. and James O.F. Comparisons of gastro-intestinal symptoms in colorectal carcinoma patients and community controls with respect to age. Gut. 1994; 35: 1267-1270.

12. Majumbdar, S.R., Fletcher, R.H. and Evans A.T. How does colorectal cancer present? Symptoms, duration, and clues to location. Amer. J. Gastroenterol. 1999; 94: 3039-3945.

13. Glass, R.L., Smith, L.E. and Cochran, R.C. Subtotal colectomy for obstructing carcinoma of the left colon. Amer. J. Surg. 1983; 145: 335-336.

14. Dunne, J.R., Gannon, C.J., Osborn, T.M., et al. Preoperative anaemia in colon cancer: assessment of risk factors. Amer. Surg. 2002; 68: 582-587.

15. Fijten, G., Starmans, R., Muris, J., et al. Predictive value of signs and symptoms for colorectal cancer in patients with rectal bleeding in general practice. Fam. Pract. 1995; 12: 279-286.

16. MacArthur, C. and Smith, A. Factors associated with speed of diagnosis, referral, and treatment in colorectal cancer. J. Epidemiol. Comm. Hlth. 1984; 38: 122-126.

17. Muris, J.W., Starmans, R., Wolfs, G.G., et al. The diagnostic value of rectal examination. Fam. Pract. 1993; 10: 34-37. 